\title{
The added value of digital breast tomosynthesis in improving diagnostic performance of BI-RADS categorization of mammographically indeterminate breast lesions
}

\author{
Mohammad Abd Alkhalik Basha* (10, Hadeer K. Safwat, Ahmed M. Alaa Eldin, Hitham A. Dawoud and Ali M. Hassanin
}

\begin{abstract}
Background: Mammographic findings are seen more clearly in tomographic images with consequent improvement of Breast Imaging Reporting and Data System (BI-RADS) in categorization of indeterminate breast lesions. This study aimed to evaluate the added value of digital breast tomosynthesis (DBT) to BI-RADS classification in categorization of indeterminate breast lesions after digital mammography (DM) as an initial approach.

Methods and results: We prospectively evaluated 296 women with BI-RADS indeterminate breast lesions (BI-RADS 0, 3, and 4) by DM between January 2018 and October 2019. All patients underwent DBT. Two radiologists evaluated lesions and assigned a BI-RADS category to each lesion according to BI-RADS lexicon 2013 classification using DM, DBT, and combined DM and DBT. The results were compared in terms of main radiological features, diagnostic performance, and BI-RADS classification using histopathology as the reference standard. A total of 355 lesions were detected on DBT and 318 lesions on DM. Thirty-seven lesions were detected by DBT and not seen by DM. The final diagnoses of 355 lesions were $58.3 \%$ benign and $41.7 \%$ malignant. In comparison to DM, DBT produced $31.5 \%$ upgrading and 35.2\% downgrading of BI-RADS scoring of breast lesions. DBT reduced number of BI-RADS 3 and 4, compared to DM. All upgraded BI-RADS 4 were malignant. The combination of DBT and DM significantly increased the performance of BI-RADS in the diagnosis of indeterminate breast lesions versus DM or DBT alone $(p<0.001)$.

Conclusion: Adding DBT to BI-RADS improves its diagnostic performance in detection and characterization of mammography indeterminate breast lesions.
\end{abstract}

Keywords: Mammography, Tomography, Breast neoplasm

\section{Key points}

- DBT produced $31.5 \%$ upgrading and $35.2 \%$ downgrading of BI-RADS scoring

- The combination of DBT and DM significantly enhanced the BI-RADS performance

- Considering added radiation dose, combined protocol could be limited to suspected lesions

* Correspondence: Mohammad_basha76@yahoo.com Department of Radiodiagnosis, Zagazig University, Zagazig, Egypt

\section{Background}

Breast cancer is the most common cancer among women in the world, accounting for about $12 \%$ of all new cancers and $27 \%$ of all female cancers [1]. Early detection becomes a critical job to reduce the morbidity and mortality associated with breast cancer [2]. Digital mammography (DM) is the primary breast imaging modality for early detection and diagnosis of breast cancer. However, some limitations persist [3]. One of the substantial limitations of DM is its use in dense breasts [4]. DM has low sensitivity and 
specificity in women with radiographically dense breast due to decrease contrast between a possible tumor and surrounding breast tissue and summation of tissues may obscure lesions [5]. Digital breast tomosynthesis (DBT) can be expected to overcome this problem by reducing or eliminating the tissue overlap. DBT technology is a modification of a DM unit to allow the acquisition of a three-dimensional (3D) volume of thin section data [4]. The role of DBT for ruling out suspected abnormalities that are identified during screening may be considered an essential diagnostic application [6]. It also allows visualization of cancers not apparent by DM [7]. The more explicit depiction with DBT should allow easier differentiation between benign and malignant lesions [4].

Breast Imaging Reporting and Data System (BIRADS) was initially developed to allow radiologists to report their level of concern that breast lesions may be missed on DM due to dense tissue but has been widely used in breast cancer research and DM performance research $[8,9]$.

Several previous studies have shown the high benefits of the addition of DBT in screening programs and the diagnostic setting [10-12]. Mammographic findings are seen more clearly in tomographic images with the consequent improvement of BI-RADS categorization. This fact is reflected, among other things, by the upgrade on BI-RADS classification of malignant lesions not correctly assessed by DM [13], in a better diagnostic performance in dense breasts with BI-RADS 0 findings [14] and in the demonstration of indeterminate lesions (BI-RADS 3 and 4) that are characterized on DM [15]. Consequently, we performed this prospective study to evaluate the added value of DBT to BI-RADS classification in categorization of indeterminate breast lesions (BIRADS 0, 3, and 4) after DM as an initial approach. Additionally, we made a simple comparison between DBT and DM to test their diagnostic performance in this context.

\section{Methods}

\section{Study design and population}

A prospective study was performed between January 2018 and October 2019. The study was approved by the research and ethical committee, and informed consent was obtained from each patient. Over the 22-month period of the study, three of the authors, who searched in the radiology information system, collected the patients who were categorized as BIRADS 0,3 , and 4 on DM consecutively to be enrolled in the study and registered the clinical, demographic, and mammographic imaging data of all patients. Inclusion criteria were (i) female $\geq 30$ years, (ii) indeterminate breast lesions by DM (BI-RADS 3 and 4), and (iii) dense breast in symptomatic patients (BI-RADS 0). The final cohort of our study included 296 female patients (mean age $46.3 \pm 9.4$ years, range 32-78 years). The patients' data are summarized in Table 1. Once enrolled, all patients were requested

\section{Table 1 Patients' data}

\begin{tabular}{|c|c|}
\hline Variable & Value \\
\hline Age, years, Mean \pm SD (range) & $46.3 \pm 9.4(19-78)$ \\
\hline \multicolumn{2}{|l|}{ Family history } \\
\hline 1st-degree relative & $89(30.1)$ \\
\hline 2nd-degree relative & $59(19.9)$ \\
\hline Negative family history & $148(50)$ \\
\hline \multicolumn{2}{|l|}{ Site of lesions } \\
\hline Right breast & $170(57.4)$ \\
\hline Left breast & $118(39.9)$ \\
\hline Both breasts & $8(2.7)$ \\
\hline \multicolumn{2}{|l|}{ Clinical presentations } \\
\hline Asymptomatic & $85(28.6)$ \\
\hline Breast lump only & $181(61.2)$ \\
\hline Breast lump with breast edema & $15(5.1)$ \\
\hline Breast lump with nipple retraction & $15(5.1)$ \\
\hline \multicolumn{2}{|l|}{ ACR BI-RADS density } \\
\hline A & $15(5.1)$ \\
\hline B & $100(33.8)$ \\
\hline C & $155(52.3)$ \\
\hline $\mathrm{D}$ & $26(8.8)$ \\
\hline \multicolumn{2}{|l|}{ Final diagnoses of 355 lesions } \\
\hline Benign & $207(58.3)$ \\
\hline Fibrocystic changes & $85(41.1)$ \\
\hline Fibroadenomas & $63(30.4)$ \\
\hline Normal & $26(12.6)$ \\
\hline Postoperative scar & $11(5.3)$ \\
\hline Granulomatous mastitis & $7(3.4)$ \\
\hline Duct ectasia & $7(3.4)$ \\
\hline Abscess & $4(1.9)$ \\
\hline Benign phylloides & $4(1.9)$ \\
\hline Malignant & $148(41.7)$ \\
\hline Invasive ductal carcinoma & $104(70.3)$ \\
\hline Invasive lobular carcinoma & $26(17.6)$ \\
\hline Ductal carcinoma in situ & $11(7.4)$ \\
\hline Mucinous carcinoma & $7(4.7)$ \\
\hline
\end{tabular}

Unless otherwise indicated, data are number with the percentage in parenthesis

$S D$ standard deviation, ACR American college of radiology, BI-RADS Breast Imaging Reporting and Data System 


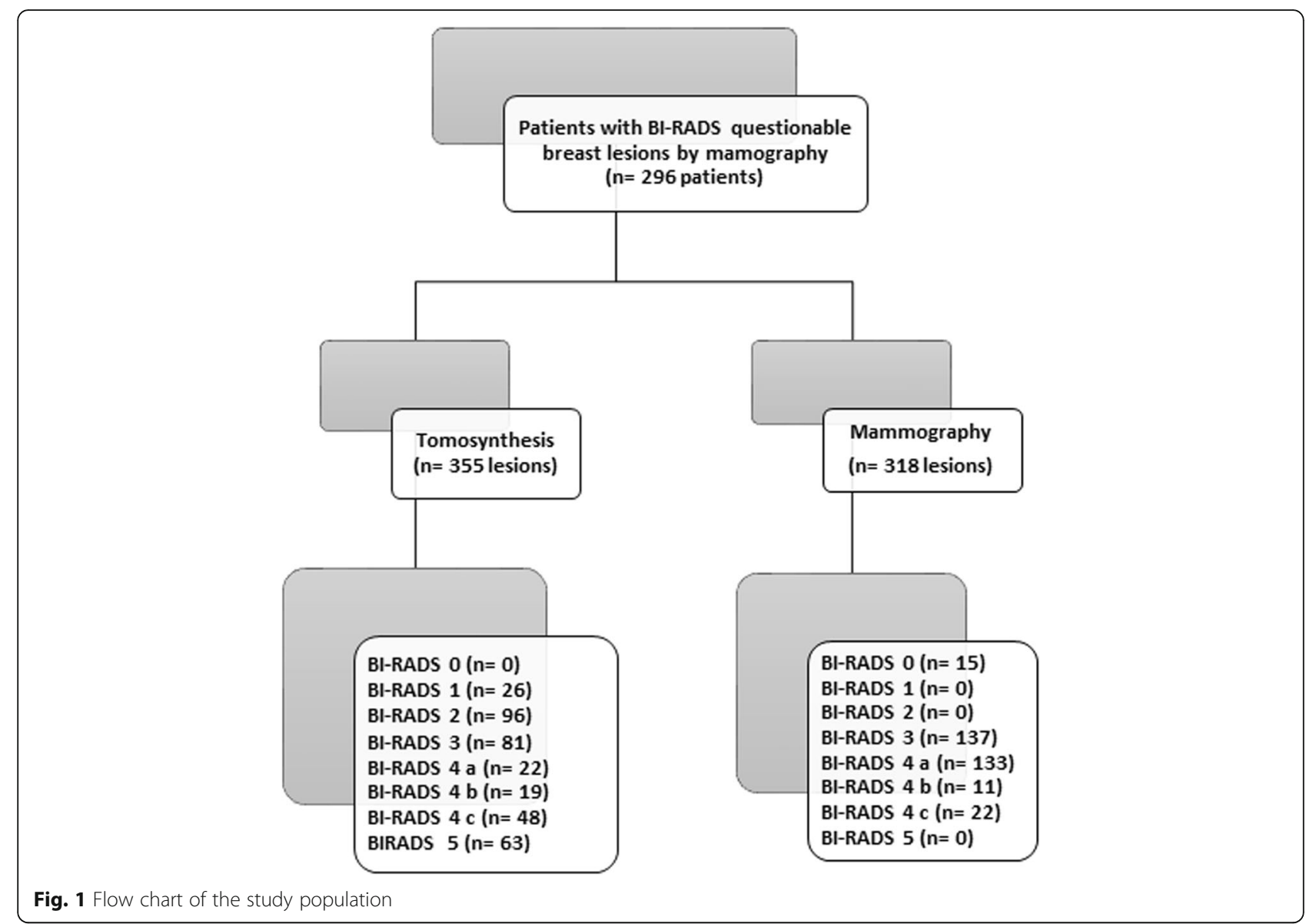

for second attendance to be subjected to DBT examinations. The flow chart of our study is illustrated in Fig. 1.

\section{The technique of mammography and tomosynthesis}

DBT examinations were performed within 2 weeks after DM examinations. All examinations were performed using a full field DM machine with 3D-DBT (Senographe Essential GE healthcare). Each breast was compressed and positioned carefully. Two views for each breast, craniocaudal (CC) and mediolateral oblique (MLO), were taken for both techniques. 3DDBT involved the acquisition of nine projections with $25^{\circ}$ scan angle. The $3 \mathrm{D}$ volume of the compressed breast was reconstructed from the $2 \mathrm{D}$ projections in the form of a series of images (slices) through the entire breast. Images from both techniques were sent to liquid-crystal display (LCD) screens for reading. No additional views were needed as further processing can be done while viewing the digital images on LCD panels such as zooming, changing contrast, brightness, darkness, inverting the background, and other processing to facilitate lesion detection.

\section{Image analysis}

The previous DM images and the DBT images were transported to the workstation for assessment. The DM and DBT images were separated for interpretation (i.e., the images for DM were interpreted without knowledge of the DBT findings). Two radiologists with 10 years of experience in breast imaging independently reviewed the DM images. After 1 month, the same two radiologists independently reviewed the DBT images. After another month, the same two radiologists independently reviewed DM and DBT images together. Any discrepancies in interpretation were resolved by a third radiologist with over 15 years of experience in breast imaging. The 1-month interval was to diminish the radiologists' memory bias. All radiologists were blinded to any clinical data or the results of the pathology. The following features obtained at DM and DBT were individually evaluated in each patient: (i) breast density, (ii) site of the lesion, (iii) size of the lesion, (iv) type of lesion (mass or focal asymmetry), (v) mass characters (shape, margin, and density), (vi) asymmetry (simple, focal, global, or developing), (vii) calcifications (morphology and 
distribution), and (viii) any other suspicious abnormalities.

The four categories of the American College of Radiology (ACR) BI-RADS scale were used to measure mammographic density [16]. The radiologists were requested to assign the BI-RADS category to all detected lesions in each of the two imaging modalities individually according to the BI-RADS lexicon 2013 classification [17]. Finally, each breast lesion had three independent BI-RADS categories (one by DM, one by DBT, and one by combined DM and DBT). In the combined protocol, the radiologists assigned a BI-RADS category based on the combined radiological features from each modality. A feature was considered positive when it was seen in at least one of DM or DBT. The results of DM and DBT for each patient were compared in terms of main radiological features, BI-RADS classification, and diagnostic performance.

\section{Reference standard}

The definitive diagnosis was validated based on histopathologic findings after US-guided biopsy $(n=221$ patients), stereotactic biopsy ( $n=32$ patients), and surgical mastectomy ( $n=43$ patients). All specimens were examined by two experienced pathologists, and the final results were acquired by consensus. Biopsies were performed to determine the lesion type by the requesting clinician.

\section{Statistical analysis}

Statistical analysis was done using SPSS software version 25 (IBM, 2017). Data were presented in tables and figures. Continuous data were presented as mean and standard deviation. Qualitative data were presented as frequencies and proportions. Pearson's chisquare $\left(x^{2}\right)$ test was used to analyze qualitative data. McNemar test was used to analyze paired qualitative data. The diagnostic performance of DM, DBT, and combined DM and DBT was estimated on a lesionbased analysis. The receiver operating characteristic (ROC) curve analysis was applied to detect the areas under the curve (AUCs). A $p$ value of $\leq 0.05$ was accepted as statistically significant.

\section{Results}

\section{Study population}

We performed our study on 296 female patients. Every enrolled patient had at least one breast lesion categorized as BI-RADS 0,3 , and 4 on DM. All patients were submitted to DBT. We detected a total of 318 lesions on DM and 355 lesions on DBT. The final diagnoses of 355 lesions were 207 (58.3\%) benign and 148 (41.7\%) malignant. The most common benign lesion was fibrocystic changes (41.1\%), and the most common malignant lesion was invasive ductal carcinoma (70.3\%). According to the ACR BI-RADS lexicon for breast density, our patients were divided into four categories: BI-RADS density A, 15 (5.1\%) patients; BI-RADS density B, 100 (33.8\%); BIRADS density C, 155 (52.3\%); and BI-RADS density D, $26(8.8 \%)$.

\section{DM and DBT findings}

The DM and DBT findings are presented in Table 2. The DM detected 318 lesions; 148 of them were mass, and 170 were non-mass, while DBT detected 355 lesions; 281 of them were mass, and 74 were non-mass. Of the 26 architectural distortions on DM, 11 revealed underlying masses on DBT. Of the 18 micro-calcifications on DM, 11 revealed underlying masses on DBT. On DBT, five out of 26 cases revealed superposition of normal glandular tissue which were wrongly diagnosed as masses on DM. Thirty-seven lesions were detected by DBT and could not be detected by DM. These lesions were found in the dense breast (BI-RADS density $\mathrm{C}$ and D) $(n=33)$ more than non-dense breast (BI-RADS density A and B) $(n=4)$.

\section{Assignment of BI-RADS category of breast lesions by DM and DBT}

The BI-RADS scoring of breast lesions is summarized in Table 3. The change in individual patient breast lesion owing to DBT, compared to DM, is presented in Table 4. In comparison to DM, DBT produced $31.5 \%$ $(112 / 355)$ upgrading of BI-RADS scoring (4.2\% (15/ $355)$ in BI-RADS $0,7.3 \%(26 / 355)$ in BI-RADS 3, $18.8 \%(56 / 355)$ in BI-RADS 4a, 3.1\% (11/355) in BIRADS $4 \mathrm{~b}$, and $1.1 \%(4 / 355)$ in BI-RADS $4 \mathrm{c})$ and $35.2 \%(125 / 355)$ downgrading of BIRADS scoring (18.9\% (67/355) in BI-RADS 3, and 16.3\% (58/355) in BI-RADS 4 a). Ninety-three (83\%) of upgraded were malignant, and 118 (94\%) of downgraded were benign. Sixty of BI-RADS $4(36.1 \%(60 / 166))$ were upgraded to BI-RADS 5 by DBT. All upgraded BI-RADS 4 were malignant, and seven of downgraded were malignant. DBT reduced the number of BI-RADS 3 and BIRADS 4 (81 and 89, respectively), compared to DM (137 and 166, respectively).

Diagnostic performance of DM and combined DM + DBT On a lesion-based analysis, the diagnostic performance of DM, DBT, and combined DBT and DM for breast cancer diagnosis is summarized in Table 5 . We considered a combination of BI-RADS 4 and 5 as conclusive for breast cancer diagnosis because the combined BI-RADS 4 and 5 produced higher levels of diagnostic performance than BI-RADS 5 alone. Based on the BI-RADS version 2013, our study showed that 
Table 2 DM and DBT findings

\begin{tabular}{|c|c|c|}
\hline Findings & $\mathrm{DM}(n=318)$ & $\mathrm{DBT}(n=355)$ \\
\hline \multicolumn{3}{|l|}{ Site of lesion } \\
\hline Right breast & $181(56.9)$ & $204(57.5)$ \\
\hline Left breast & $122(38.4)$ & $136(38.3)$ \\
\hline Both breasts & $15(4.7)$ & $15(4.2)$ \\
\hline \multicolumn{3}{|l|}{ Type of lesion } \\
\hline Mass & $148(46.5)$ & $281(79.2)$ \\
\hline Asymmetry & $111(34.9)$ & \\
\hline Architecture distortion & $26(8.2)$ & $15(4.2)$ \\
\hline Clusters of micro-calcification with no underlying mass & $18(5.7)$ & $7(2)$ \\
\hline Dense breast (BIRADS 0) & $15(4.7)$ & \\
\hline Dilated ducts & & $11(3.1)$ \\
\hline Asymmetrical densities & & $15(4.2)$ \\
\hline Overlapped glandular tissue & $21(6.6)$ & $26(7.3)$ \\
\hline \multicolumn{3}{|l|}{ Characters of mass } \\
\hline \multicolumn{3}{|l|}{ Mass margin } \\
\hline Obscured on mammography and speculated on tomosynthesis & $70(47.3)$ & $111(39.5)$ \\
\hline III-defined & $56(37.8)$ & $44(15.7)$ \\
\hline Well-defined & $22(14.9)$ & $126(44.8)$ \\
\hline \multicolumn{3}{|l|}{ Mass shape } \\
\hline Irregular & $67(45.3)$ & $130(46.3)$ \\
\hline Round & $44(29.7)$ & $92(32.7)$ \\
\hline Oval & $19(12.8)$ & $37(13.2)$ \\
\hline Macrolobulated & $18(12.2)$ & $22(7.8)$ \\
\hline \multicolumn{3}{|l|}{ ACR BI-RADS density } \\
\hline A & $15(4.7)$ & $15(4.2)$ \\
\hline B & $107(33.7)$ & $111(31.3)$ \\
\hline C & $170(53.5)$ & $189(53.2)$ \\
\hline $\mathrm{D}$ & $26(8.1)$ & $40(11.3)$ \\
\hline
\end{tabular}

The data are represented as numbers with the corresponding percentages given in parentheses

$D B T$ digital breast tomosynthesis, DM digital mammography, BI-RADS Breast Imaging Reporting and Data System, ACR American college of radiology

Table 3 BI-RADS categories of the 355 breast lesions detected on DBT and DM in relation to the final diagnosis

\begin{tabular}{|c|c|c|c|c|c|c|}
\hline & \multicolumn{3}{|l|}{ DBT } & \multicolumn{3}{|l|}{$\mathrm{DM}$} \\
\hline & Malignant & Benign & Total & Malignant & Benign & Total \\
\hline Not seen & 0 & 0 & 0 & $15(4.2)$ & $22(6.2)$ & $37(10.4)$ \\
\hline BI-RADS 0 & 0 & 0 & 0 & $4(1.1)$ & $11(3.1)$ & $15(4.2)$ \\
\hline BI-RADS 1 & 0 & $26(7.3)$ & $26(7.3)$ & 0 & 0 & 0 \\
\hline BI-RADS 2 & 0 & $96(27)$ & $96(27)$ & 0 & 0 & 0 \\
\hline BI-RADS 3 & $16(4.5)$ & 65 (18.3) & 81 (22.8) & $30(8.5)$ & 107 (30.1) & 137 (38.6) \\
\hline BI-RADS 4 a & $16(4.5)$ & $6(1.8)$ & $22(6.3)$ & 66 (18.6) & 67 (18.9) & $133(37.5)$ \\
\hline BI-RADS 4 b & $19(5.4)$ & 0 & $19(5.4)$ & $11(3.1)$ & 0 & $11(3.1)$ \\
\hline BI-RADS $4 \mathrm{c}$ & $34(9.6)$ & $14(3.9)$ & 48 (13.5) & $22(6.2)$ & 0 & $22(6.2)$ \\
\hline BI-RADS 5 & 63 (17.7) & 0 & $63(17.7)$ & 0 & 0 & 0 \\
\hline Total & $148(41.7)$ & $207(58.3)$ & $355(100)$ & $148(41.7)$ & $207(58.3)$ & $355(100)$ \\
\hline
\end{tabular}

The data are represented as numbers with the corresponding percentages given in parentheses 
Table 4 Change in individual breast lesion grading on account of DBT, compared to DM

\begin{tabular}{|c|c|c|c|c|c|c|c|c|c|}
\hline \multirow[t]{2}{*}{$\mathrm{DM}$} & \multicolumn{9}{|l|}{ DBT } \\
\hline & BI-RADS 0 & BI-RADS 1 & BI-RADS 2 & BI-RADS 3 & BI-RADS 4 a & BI-RADS $4 \mathrm{~b}$ & BI-RADS $4 \mathrm{c}$ & BI-RADS 5 & Total \\
\hline Not seen & & & $19(5.4)$ & $4(1.1)$ & & $7(2)$ & $4(1.1)$ & $3(0.85)$ & $37(10.4)$ \\
\hline BI-RADS 0 & & & & $11(3.1)$ & & & $4(1.1)$ & & $15(4.2)$ \\
\hline BI-RADS 1 & & & & & & & & & 0 \\
\hline BI-RADS 2 & & & & & & & & & 0 \\
\hline BI-RADS 3 & & $26(7.3)$ & $41(11.5)$ & $44(12.4)$ & $3(0.85)$ & $12(3.4)$ & $11(3.1)$ & & $137(38.6)$ \\
\hline BI-RADS 4 a & & & $36(10.1)$ & $22(6.2)$ & $195.4)$ & & $11(3.1)$ & $45(12.7)$ & $133(37.5)$ \\
\hline BI-RADS 4 b & & & & & & & & $11(3.1)$ & $11(3.1)$ \\
\hline BI-RADS $4 \mathrm{c}$ & & & & & & & $18(5.1)$ & $4(1.1)$ & $22(6.2)$ \\
\hline BI-RADS 5 & & & & & & & & & 0 \\
\hline Total & & $26(7.3)$ & $96(27)$ & $81(22.8)$ & $22(6.3)$ & $19(5.4)$ & 48 (13.5) & $63(17.7)$ & 355 (100) \\
\hline
\end{tabular}

The data are represented as numbers with the corresponding percentages given in parentheses

The different colors indicate whether DBT upgraded (yellow), downgraded (blue), or kept the grade the same (green) as DM

DBT digital breast tomosynthesis, DM digital mammography, BI-RADS Breast Imaging Reporting and Data System

BI-RADS with DBT yielded significantly higher accuracy, sensitivity, and specificity than BI-RADS with $\mathrm{DM}$ in the diagnosis of breast cancer $(p<0.001)$. The combination of DBT and DM significantly increased the performance of BI-RADS in the diagnosis of breast cancer versus DM or DBT alone $(p<0.001)$. $\mathrm{DM}$ had more false-positive and false-negative rates than DBT.

\section{ROC analyses}

We analyzed the data set of the diagnostic performance of BI-RADS with DM, BI-RADS with DBT, and BI-RADS with combined DM and DBT for breast cancer diagnosis using the ROC curve. When the ROC areas were compared, it was found that BIRADS with DBT was significantly superior to BIRADS with DM in breast cancer diagnosis (AUC:
0.883 vs. $0.619 ; p<0.0001 ; 95 \%$ CI 0.214 to 0.313$)$, and the BI-RADS with combined DM and DBT was significantly superior to BI-RADS with DM or BIRADS with DBT alone (AUC: 0.971; $p<0.0001 ; 95 \%$ CI 0.0565 to 0.120 ) (Fig. 2).

Our study's representative cases are illustrated in Figs. 3,4 , and 5 .

\section{Discussion}

In this study, we mainly focused on indeterminate BIRADS categories, because reducing BI-RADS 0,3 , and 4 has pivotal implications for patient care. We aimed to display the added value of DBT to the BI-RADS classification. Few studies evaluated this topic; most of them have investigated category $3[18,19]$, and some have also included category 0 [20]. However, this study focused exclusively on BI-RADS 0,3 , and 4

Table 5 The diagnostic performance of BI-RADS with mammography, BI-RADS with tomosynthesis, and BI-RADS with combined tomosynthesis and mammography for confident breast cancer diagnosis considered BI-RADS 4 and 5 as predictive of malignancy

\begin{tabular}{|c|c|c|c|c|c|c|}
\hline \multirow[t]{2}{*}{ Criterion } & \multicolumn{2}{|c|}{ Mammography } & \multicolumn{2}{|c|}{ Tomosynthesis } & \multicolumn{2}{|c|}{ Combined } \\
\hline & $\%$ & $95 \% \mathrm{Cl}$ & $\%$ & $95 \% \mathrm{Cl}$ & $\%$ & $95 \% \mathrm{Cl}$ \\
\hline Accuracy & 67.3 & & 90 & & 97.5 & \\
\hline Sensitivity & 66.9 & 58.7 to 74.4 & 89.2 & 83 to 93.7 & 98.7 & 95.2 to 99.8 \\
\hline Specificity & 67.6 & 60.8 to $74 \%$ & 90.3 & 85.5 to 94 & 96.6 & 93.2 to 98.6 \\
\hline Positive likelihood ratio & 2.1 & 1.7 to 2.6 & 9.23 & 6.1 to 14.1 & 29.2 & 14.1 to 60.4 \\
\hline Negative likelihood ratio & 0.49 & 0.38 to 0.63 & 0.12 & 0.08 to 0.19 & 0.01 & 0 to 0.06 \\
\hline Disease prevalence & 41.7 & 36.5 to 47 & 41.7 & 36.5 to 47 & 41.7 & 36.5 to 47 \\
\hline PPV & 59.6 & 51.8 to 67.2 & 86.8 & 80.4 to 91.8 & 95.4 & 90.8 to 98.1 \\
\hline NPV & 74.1 & 67.2 to 80.2 & 92.1 & 87.5 to 95 & 99 & 96.5 to 99.9 \\
\hline
\end{tabular}

BI-RADS Breast Imaging Reporting and Data System, AUC area under curve, $P P V$ positive predictive value, $N P V$ negative predictive value, $C I$ confidence interval 


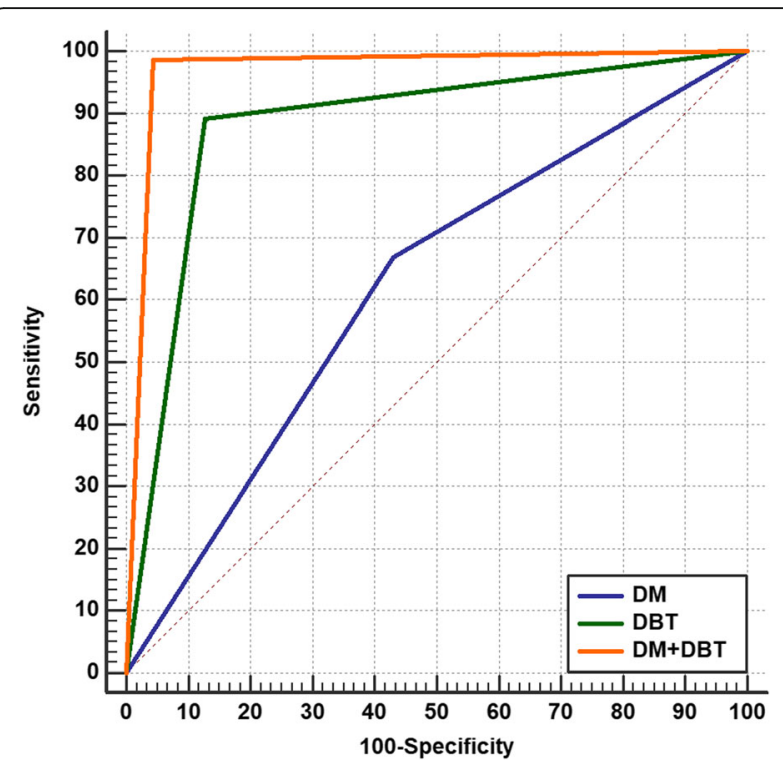

Fig. 2 Comparison of the ROC areas of BI-RADS with DM, BI-RADS with DBT, and BI-RADS with combined DM and DBT for breast cancer diagnosis as evidenced by histopathology as the reference standard

categories. The overall results of our study confirmed the high diagnostic performance of DBT in the evaluation of indeterminate BI-RADS categories. We found that combined DBT and DM in BI-RADS resulted in a superior sensitivity (98.7\%), specificity (96.6\%), and accuracy $(97.5 \%)$ for indeterminate breast lesion categorization than DM or DBT alone; the sensitivity, specificity, and accuracy declined to $66.9 \%, 67.6 \%$, and $67.3 \%$, respectively, for the DM assessment and $89.2 \%$, $90.3 \%$, and $90 \%$, respectively, for the DBT assessment. Moreover, we found that DBT had a significantly higher sensitivity, specificity, and accuracy than DM in the diagnosis of indeterminate breast lesions. The above findings are matching with that of many previous studies $[10,12,19,21-25]$, which have established that DBT increases the sensitivity and specificity of DM. Consequently, in light of our data, and considering the high diagnostic performance of DBT, we recommend the use of DBT as an additional imaging modality to improve diagnostic accuracy in detecting and characterizing indeterminate breast lesions.

We found that DBT produced a significant change of BI-RADS category in $66.7 \%$ lesions with an upgrade in $31.5 \%$ lesions ( $83 \%$ were malignant) and a downgrade in $35.2 \%$ lesions (94\% were benign) in comparison to the DM. This finding agrees with the recent study published by Raghu et al. [12] who have proved that the addition of DBT has been found to change rates of BI-RADS final assessment over time. Similarly, Michell et al. [26] showed a reduction of probably benign cases in $57.8 \%$ by an additional DBT.

A remarkable observation of our study was the higher number of lesions identified with DBT than with DM [37 (10.4\%)], with BI-RADS 2 lesion representing the greatest number of these missed lesions on DM [19 (51.4\%)]. We found that the main cause for missing a lesion on DM was poor visibility due to dense breast parenchyma, tissue overlap, and a radiographically non-conspicuous lesion. In contrast, the DBT decreased interference from overlapping breast tissue and improved lesion conspicuity. These missed lesions on DM cause a significant upgrade of the BIRADS categories between DM and DBT and subsequently increased diagnostic performance of DBT over DM. This finding indicates that DBT is more accurate than DM in the identification of breast lesion, which is comparable to the previous findings [10, 15 , 19]. The increased number of lesions detected on DBT is most probably due to the use of rebuilt images in DBT, as stated by Andersson et al. [13]. These images are obtained from different angles from the breast in a short scanning process and allow the assessment of breast parenchyma where lesions may go unnoticed or less evident due to tissue overlap or increased breast density.

The reduction in the number of BI-RADS 3 and 4 lesions is one of the potential advantages of DBT as some lesions that were categorized as BI-RADS 3 and 4 on DM was upgraded to BI-RADS 5 or downgraded to BIRADS 1 and 2 based on DBT. This increase in the identification of BI-RADS 3 and 4 lesions by DBT likely result in reduced follow-up of lesions that would not have been identified by DM alone and diminished the requirement for biopsy. These results are comparable to those reported by previous studies [13, 27, 28].

Although DBT has better diagnostic performance than DM, still some breast lesions could not be determined on DBT. On DBT images, improved visualization of a partially or totally smooth boundary in some malignant masses may potentially lead to a misdiagnosis that is false benign diagnosis [29]. Thirty-six of the breast lesions in this study were misdiagnosed on DBT (20 false-positives and 16 falsenegatives). Sixteen masses were described as probably benign masses on DBT, but histopathology revealed breast cancer. However, the misdiagnosed lesions on DBT were less than that on DM (67 false-positives and 49 false-negatives). The combined DM and DBT decreased misdiagnosed lesions (seven false-positives and two false-negatives) when compared to DM or DBT alone. Accordingly, our study recommends the 

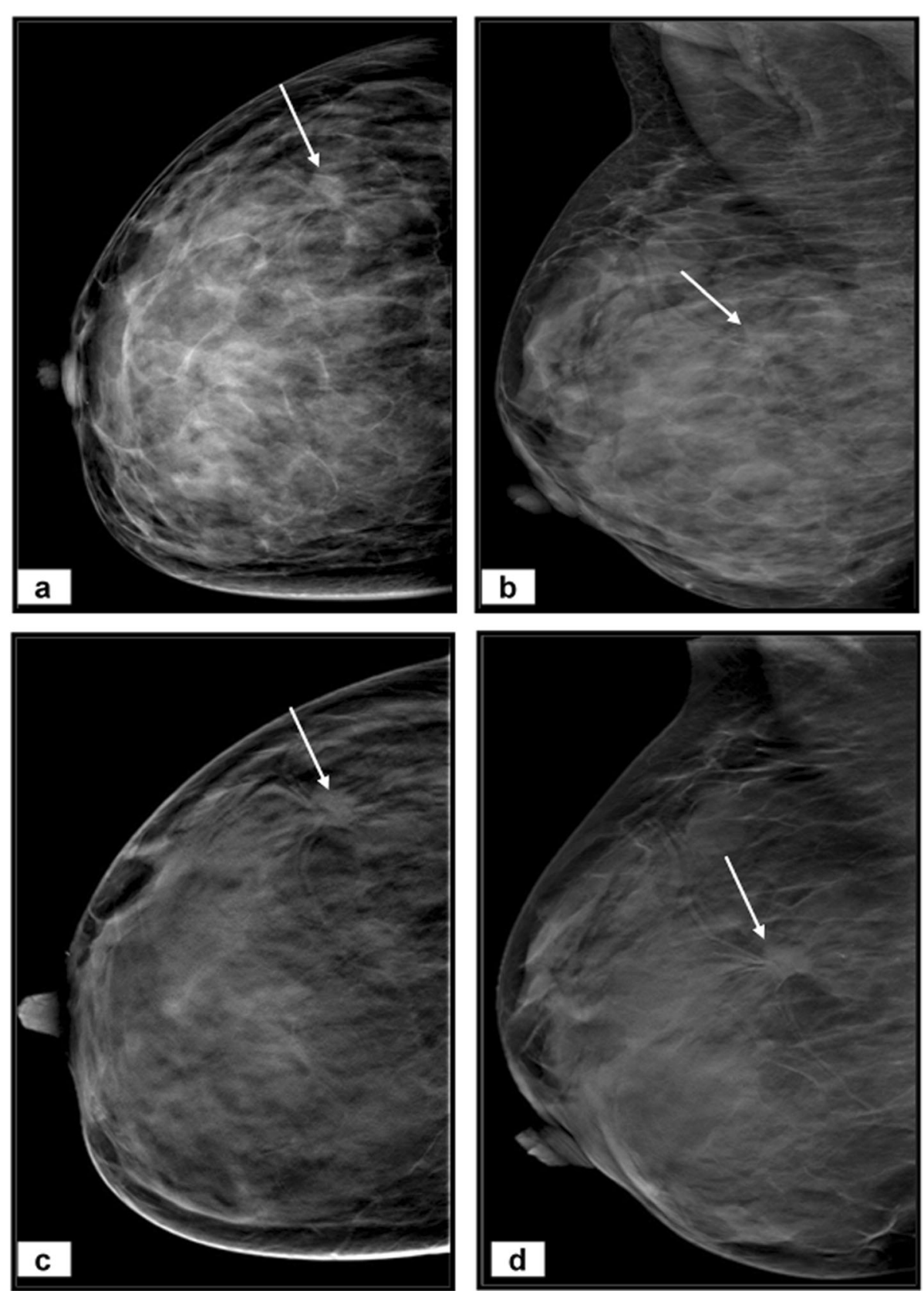

Fig. 3 A 35-year-old woman complains of a right breast lump. a Craniocaudal and (b) mediolateral oblique DM images of right breast reveal extremely dense breast (BI-RADS D) with an outer central area of architectural distortion (arrows). No spiculated masses or microcalcifications. c Craniocaudal and (d) mediolateral oblique DBT images show a definite lesion with spiculated margins and measures $27 \times 25 \mathrm{~mm}$ (arrows). The lesion was categorized as BI-RADS 4a by DM and BI-RADS 4c by DBT. Histopathology after surgery revealed invasive lobular carcinoma

use of BI-RADS with a combined DM and DBT protocol, as it improved the BI-RADS performance for diagnosis of indeterminate breast lesions with subsequent potentially better disease management. Similar findings have been seen in various other studies [30-32], in which the addition of DBT decrease in the number of false cases.
When comparing the ROC areas, it was found that DBT is significantly superior to DM in breast cancer diagnosis (AUC $=0.883$ vs $0.619 ; p<0.0001$ ), and the BI-RADS with combined DM and DBT was significantly superior to BI-RADS with DM or BI-RADS with DBT alone (AUC: 0.971; $p<0.0001$ ). Similarly, Cai et al. [33] analyzed 79 cases with pathologic results by using ROC 

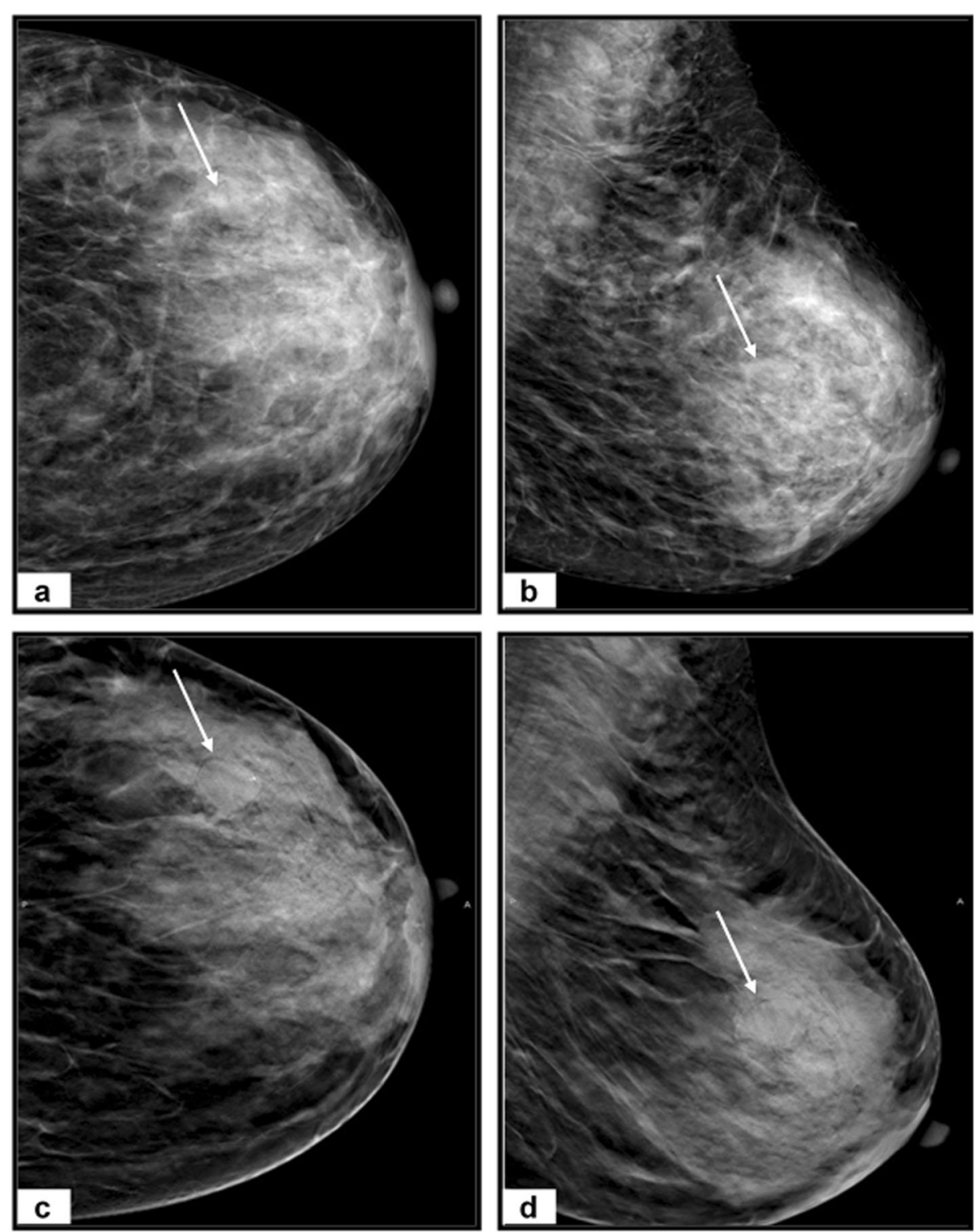

Fig. 4 A 48-year-old woman complains of a left breast lump. a Craniocaudal and (b) mediolateral oblique DM images of left breast reveal heterogeneous dense breast (BI-RADS C) with upper outer quadrant dense lesion with obscured margin (arrows). No spiculated masses or microcalcifications. c Craniocaudal and (d) mediolateral oblique DBT images show a well-defined rounded medium dense lesion with smooth margins and minute peripheral calcific foci, measures $16 \times 16 \mathrm{~mm}$ and associated with the characteristic halo sign (arrows). The lesion was categorized as BI-RADS 4C by DM and BI-RADS 2 by DBT. Histopathology after US-guided biopsy revealed simple cyst

curve and showed that the AUC of the combined DM and DBT was greater than that of DM alone (0.914 vs. 0.805). Also, Thomassin et al. [34] calculated AUC by averaging the ROC from four readers; the mean AUC for BI-RADS with combined DM and DBT was higher than that calculated for BI-RADS with DM alone $(0.809$ vs. $0.685 ; p<0.01)$. In contrast, Gennaro et al. [35] concluded that the overall clinical performance with DBT and DM for malignant versus all other cases was not significantly different (AUCs $=0.851$ vs. $0.836, p=0.645$ ).
Finally, our study demonstrates that BI-RADS could diagnose the same patient with breast lesions by DBT but not by DM. Thus, we should use DBT in BI-RADS categorization of breast lesions as if we used DM alone as decisive for breast cancer diagnosis; a significant number of breast cancer lesions would be missed by the BI-RADS. The suggested approach for the evaluation of breast cancer lesions according to our results is as follows: first, we perform the DM as a primary imaging modality; if the BI-RAD category provides a confident 

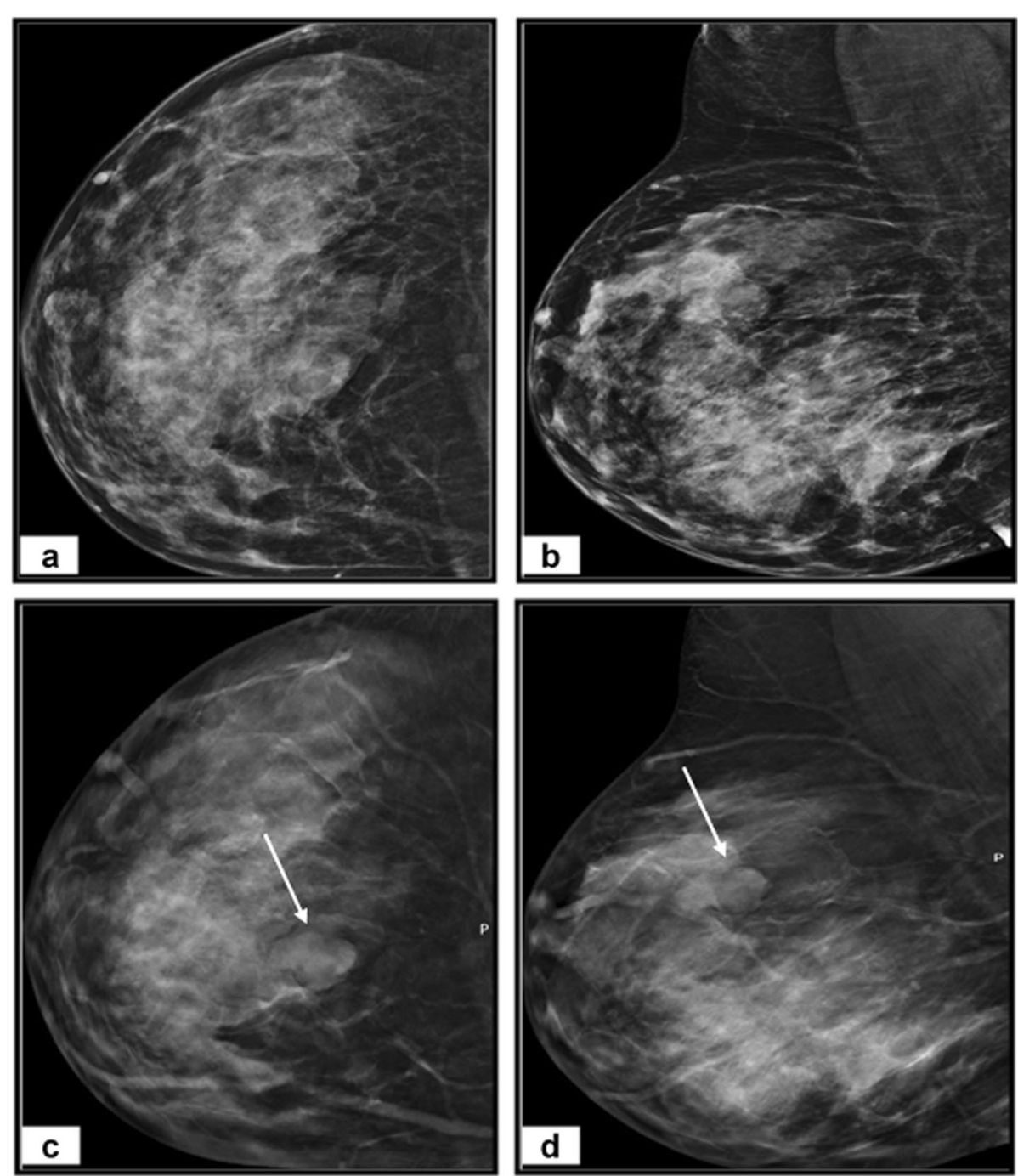

Fig. 5 A 36-year-old woman complains of a right breast lump. a Craniocaudal and (b) mediolateral oblique DM images of left breast reveal heterogeneous dense breast (BI-RADS C). The DM images are inconclusive and need further assessment. c Craniocaudal and (d) mediolateral oblique DBT images show upper inner quadrant medium density oval-shaped lesion with macrolobulated margins and measures $22 \times 20 \mathrm{~mm}$ (arrows). The lesion was categorized as BI-RADS 0 by DM and BI-RADS III by DBT. Histopathology after US-guided biopsy revealed fibroadenoma

diagnosis of being benign or malignant (i.e., BI-RADS 1, 2 , or 5), the patients go on to the management without further additional imaging. In cases of indeterminate diagnosis (i.e., BI-RADS 0, 3, or 4), we perform DBT, and the BI-RADS category is determined by the combination of DM and DBT findings. We perform the DM initially and not DBT as the former's lower cost and wide availability.

There are some limitations to our study. First, we focused on indeterminate breast lesions (BI-RADS 0, 3, and 4) and did not consider other BI-RADS categories (BI-RADS 1, 2, and 5). Second, no trial was conducted to analyze the inter-reader agreement in the classification of breast lesions. Third, we did not address the
DBT performance in each breast density category. Fourth, the cost-effectiveness and the added radiation dose of combined DM and DBT protocol may be disadvantages of this protocol. Thus, we suggest that the combined protocol be limited to doubted lesions when there remains uncertainty in the BI-RADS category after conducting DM alone.

\section{Conclusion}

Adding DBT to BI-RADS classification improves its diagnostic performance in the detection and characterization of lesions categorized as BI-RADS 0,3 , and 4 by DM. 


\section{Abbreviations}

3D: Three-dimensional; ACR: American College of Radiology; AUC: Area under the curve; BI-RADS: Breast Imaging Reporting and Data System; Cl: Confidence interval; DBT: Digital breast tomosynthesis; DM: Digital mammography; LCD: Liquid-crystal display; ROC: Receiver operating characteristic

\section{Acknowledgment}

The authors thank all staff members and colleagues in radiology departments-Zagazig University and Cairo National Cancer Institute for their helpful cooperation and all the study participants for their patience and support.

\section{Ethical approval and consent to participate}

Institutional review board approval was obtained. Written informed consent was obtained from all patients.

\section{Authors' contributions}

Guarantor of integrity of the entire study-MB. Study concepts and design-MB, AH, and HS. Literature research-HD and AA. Clinical studies- $-\mathrm{HS}, \mathrm{AH}, \mathrm{HS}$, and $\mathrm{AA}$. Experimental studies/data analysis- $\mathrm{MB}, \mathrm{AH}$, and HS. Statistical analysis-MB. Manuscript preparation-MB and AH. Manuscript editing- $\mathrm{MB}$ and $\mathrm{AH}$. All authors read and approved the final manuscript.

\section{Funding}

The authors declare that this work has not received any funding.

\section{Availability of data and materials}

The datasets used and/or analyzed during the current study are available from the corresponding author on reasonable request.

\section{Consent for publication}

Not applicable.

\section{Competing interests}

The authors declare that they have no competing interests.

Received: 19 November 2019 Accepted: 13 January 2020

Published online: 14 February 2020

\section{References}

1. Siegel RL, Miller KD, Jemal A (2016) Cancer statistics, 2016. CA Cancer J Clin 66:7-30

2. Dey S (2014) Preventing breast cancer in LMICs via screening and/or early detection: The real and the surreal. World J Clin Oncol 5:509-519

3. van den Biggelaar FJ, Kessels AG, van Engelshoven JM, Flobbe K (2009) Strategies for digital mammography interpretation in a clinical patient population. Int J Cancer 125:2923-2929

4. Park JM, Franken EA Jr, Garg M, Fajardo LL, Niklason LT (2007) Breast tomosynthesis: Present considerations and future applications. Radiographics 27:S231-S240

5. Fallenberg EM, Dromain C, Diekmann F et al (2014) Contrast-enhanced spectral mammography versus MRI: initial results in the detection of breast cancer and assessment of tumour size. Eur Radiol 24:256-264

6. Gur D (2007) Tomosynthesis: potential clinical role in breast imaging. AJR Am J Roentgenol 189:614-615

7. Helvie M (2010) Digital mammography imaging: breast tomosynthesis and advanced applications. Radiol Clin North Am 48:917-929

8. Yaffe MJ (2008) Mammographic density — measurement of mammographic density. Breast Cancer Res 10:209.

9. Smith-Bindman R, Chu P, Miglioretti DL et al (2005) Physician predictors of mammographic accuracy. J Natl Cancer Inst 97:358-367

10. Skaane P, Bandos Al, Gullien R et al (2013) Comparison of digital mammography alone and digital mammography plus tomosynthesis in a population-based screening program. Radiology 267:47-56

11. Zuley ML, Bandos Al, Ganott MA et al (2013) Digital breast tomosynthesis versus supplemental diagnostic mammographic views for evaluation of noncalcified breast lesions. Radiology 266:89-95
12. Raghu M, Durand MA, Andrejeva L et al (2016) Tomosynthesis in the diagnostic setting: changing rates of BI-RADS final assessment over time. Radiology 281:54-61

13. Andersson I, Ikeda DM, Zackrisson S et al (2008) Breast tomosynthesis and digital mammography: a comparison of breast cancer visibility and BIRADS classification in a population of cancers with subtlemammographic findings. Eur Radiol 18:2817-2825

14. Lee WK, Chung J, Cha ES, Lee JE, Kim JH (2016) Digital breast tomosynthesis and breast ultrasound: Additional roles in dense breasts with category 0 at conventional digital mammography. Eur J Radiol 85:291-296

15. Ray KM, Turner E, Sickles EA, Joe BN (2015) Suspicious findings at digital breast tomosynthesis occult to conventional digital mammography: imaging features and pathology Findings. Breast J 21:538-5342

16. American College of Radiology (2013) Breast imaging reporting and data system, 5th edn. ACR, Reston, VA

17. D'Orsi CJ, Sickles EA, Mendelson EB et al (2013) ACR BI-RADS ${ }^{\circledR}$ At-las, Breast imaging reporting and data system, 5th edn. ACR, Reston, VA

18. Stepanek T, Constantinou N, Marshall H et al (2019) Changes in the utilization of the BI-RADS Category 3 assessment in recalled patients before and after the implementation of screening digital breast tomosynthesis. Acad Radiol 26:1515-25

19. Bahrs SD, Otto V, Hattermann V et al (2018) Breast tomosynthesis for the clarification of mammographic BI-RADS 3 lesions can decrease follow-up examinations and enables immediate cancer diagnosis. Acta Radiol 59: $1176-1183$

20. Emlik GD, Poyraz N, Altunkeser A (2017) Digital breast tomosynthesis and ultrasonography: diagnostic performance and effect on recall rates versus digital mammography in category 0. Int J Clin Exp Med 10:10668-10675

21. Haas BM, Karla V, Geisel J et al (2013) Comparison of tomosynthesis plus digital mammography and digital mammography alone for breast cancer screening. Radiology 269:694-700

22. Waldherr C, Gerny P, Altermatt HJ et al (2013) Value of one-view breast tomosynthesis versus two-view mammography in diagnostic workup of women with clinical signs and symptoms and in women recalled from screening. AJR Am J Roentgenol 200:226-231

23. Poplack SP, Tosteson TD, Kogel CA et al (2007) Digital breast tomosynthesis: initial experience in 98 women with abnormal digital screening mammography. AJR Am J Roentgenol 189:616-623

24. Gennaro G, Toledano A, Di Maggio C et al (2010) Digital breast tomosynthesis versus digital mammography: a clinical performance study. Eur Radiol 20:1545-1553

25. Rafferty EA, Park JM, Philpotts LE et al (2013) Assessing radiologist performance using combined digital mammography and breast tomosynthesis compared with digital mammography alone: results of a multicenter, multireader trial. Radiology 266:104-113

26. Michell MJ, Iqbal A, Wasan RK et al (2012) A comparison of the accuracy of film-screen mammography, full-field digital mammography, and digital breast tomosynthesis. Clin Radiol 67:976-981

27. Rose SL, Tidwell AL, Ice MF, Nordmann AS, Sexton R Jr, Song R (2014) A reader study comparing prospective tomosynthesis interpretations with retrospective readings of the corresponding FFDM examinations. Acad Radiol 21:1204-1210

28. Galati F, Marzocca F, Bassetti E et al (2047) (2017) Added value of digital breast tomosynthesis combined with digital mammography according to reader agreement: changes in BI-RADS rate and follow-up management. Breast Care (Basel) 12:218-222

29. Nakashima K, Uematsu T, Itoh T et al (2017) Comparison of visibility of circumscribed masses on Digital Breast Tomosynthesis (DBT) and 2D mammography: are circumscribed masses better visualized and assured of being benign on DBT? Eur Radiol 27:570-577

30. Haas BM, Kalra V, Geisel J, Raghu M, Durand M, Philpotts LE (2013) Comparison of tomosynthesis plus digital mammography and digital mammography alone for breast cancer screening. Radiology 269:694700

31. Rose SL, Tidwell AL, Bujnoch $L$, Kushwaha AC, Nordmann AS, Sexton R Jr (2013) Implementation of breast tomosynthesis in a routine screening practice: an observational study. AJR Am J Roentgenol 200:1401-1408

32. Skaane P, Bandos Al, Gullien R et al (2013) Prospective trial comparing fullfield digital mammography (FFDM) versus combined FFDM and tomosynthesis in a population-based screening programme using independent double reading with arbitration. Eur Radiol 23:2061-2071 
33. Cai SQ, Yan JX, Chen QS, Huang ML, Cai DL (2015) Significance and application of DBT for the BI-RADS classification of breast cancer. Asian Pac J Cancer Prev 16:4109-4114

34. Thomassin-Naggara I, Perrot N, Dechoux S, Ribeiro C, Chopier J, De Bazelaire C (2015) Added value of one-view breast tomosynthesis combined with digital mammography according to reader experience. Eur J Radiol 84:235241

35. Gennaro G, Toledano A, Di Maggio C et al (2010) Digital breast tomosynthesis versus digital mammography: a clinical performance study. Eur Radiol 20:1545-1553

\section{Publisher's Note}

Springer Nature remains neutral with regard to jurisdictional claims in published maps and institutional affiliations.

\section{Submit your manuscript to a SpringerOpen ${ }^{\circ}$ journal and benefit from:}

- Convenient online submission

- Rigorous peer review

- Open access: articles freely available online

- High visibility within the field

- Retaining the copyright to your article

Submit your next manuscript at $\boldsymbol{\nabla}$ springeropen.com 